American Journal of Infectious Diseases 4 (4): 262-266, 2008

ISSN 1553-6203

(C) 2008 Science Publications

\title{
Risk Factors for Bacteremia and Predictors of Mortality of Patients with Bloodstream Infection with Methicillin-Resistant Staphylococcus aureus
}

\author{
Elcio Bakowski, Sérgio Barsantini Wey and Eduardo Alexandrino Servolo Medeiros \\ Infectious Diseases Division, Department of Medicine, Universidade Federal de São Paulo, Rua Napoleão \\ de Barros, 690/2², Vila Clementino. 04024-002, São Paulo, SP, Brazil
}

\begin{abstract}
The objective of this study was to evaluate the incidence of infection and the risk factors associated to bacteraemia and mortality due to Methicillin-Resistant Staphylococcus Aureus (MRSA). This cohort study, conducted from August 2004 through November 2005, involved 60 consecutively identified patients with bloodstream MRSA infection. The control group included 240 non-infected patients, randomly selected and hospitalized on the day, or immediately after, results of blood culture of case patients were available. A case-control (1:4) study was conducted for evaluation of risk factors for bacteraemia and death. The incidence of infection was $1.99 / 1000$ admissions. Mortality rates were $30 \%$ among patients and $5.38 \%$ among controls, with an attributed death rate of $24.6 \%$. Multivariate analysis indicated the following risk factors for bacteraemia: severity predictors McCabe $=1, \mathrm{ASA}>2$ or APACHE II>10 (OR: 4.1; 95\% CI: 1.8 - 9.4) and use of central venous catheter (OR: 7.3; 95\% CI: 2.4-22.2). University analysis showed as risk factors for mortality: age (OR: 1.08; 95\% CI: 1.03-1.12), severity predictors McCabe $=1$, ASA > 2 or APACHE II > 10 (OR: 9.13; 95\% CI: 2.57-32.35), use of mechanical ventilation (OR: 10.32; 95\% CI: 1.78-59.51) and use of corticoids (OR: 9.96; 95\% CI: 2.69-36.78). Our results when investigating risk factors for bacteremia and mortality due to MRSA showed moderate influence of control group selection, in comparison to studies in which methicillinsensitive $S$. aureus infection is used as control.
\end{abstract}

Key words: $S$. aureus, bacteraemia, risk factors, incidence, mortality

\section{INTRODUCTION}

S. aureus is an important agent of nosocomial bacterial infection. The resulting wide range of clinical manifestations suggests a pristine nature, supporting the idea that man is a natural reservoir of $S$. aureus ${ }^{[1]}$ and that the bacteria is the most common human pathogen ${ }^{[2]}$.

In the context of nosocomial infections, its importance is stressed by the fact that it is the most prevalent agent in the US, responsible for $20 \%$ of surgical site infections, $18 \%$ of pneumonias and $13 \%$ of bloodstream infections ${ }^{[3]}$. S. aureus bloodstream infection deserves special attention. The condition shows high frequency, representing for instance $12 \%$ of all nosocomial infections in an European study involving over 10,000 Intensive Care Unit (ICU) patients $^{[4]}$. It is also related to significant mortality rates $(4 \mathrm{a} 30 \%)^{[5]}$.

Methicillin-Resistant Staphylococcus Aureus (MRSA) infection, unfortunately, is not restricted to hospital environment and MRSA community outbreaks have been reported among US adults and teenagers, indians ${ }^{[6]}$, homosexual men ${ }^{[7]}$, military personnel ${ }^{[8]}$, intravenous drug users, contact sports athletes and individuals infected with the human immunodeficiency virus ${ }^{[9,10]}$. Not only soft tissues are infected and invasive and lethal infections have been observed in children ${ }^{[11]}$.

Epidemiological studies involving $S$. aureus or other agents may be designed for the investigation and identification of elements which have important role in our understanding of risk factors for infection and for its unfavourable progression leading to sequelae or even death.

What is the best type of control, in case-control studies, to identify risk factors for MRSA bacteraemia or death? Common sense indicates that resistance to antibiotics, represented here by methicillin, confers to the bacteria the capacity to be more aggressive. A metaanalysis conducted by Cosgrove et al. ${ }^{[12]}$ showed that methicillin-resistant strains were indeed related to more severe outcomes.

Corresponding Author: Eduardo Alexandrino Servolo de Medeiros - Infectious Diseases Division, Department of Medicine, Universidade Federal de São Paulo, Rua Napoleão de Barros, 690, $2^{\circ}$ andar, Vila Clementino. 04024-002, São Paulo, SP, Brazil 
It is however interesting to consider, besides methicillin resistance, which is exclusive of MRSA and not reproducible in the control groups, other elements possibly involved with mortality, including biological characteristics of $S$. aureus such as the presence of adhesins and toxins, or environmental factors such as the use of catheters or mechanical ventilation. In this kind of analysis, risk factors seem to interact originating more severe situations and increased risk of death or bacterial infection, so that the epidemiological elements conferring morbidity to $S$. aureus could also be present in cases of infection by other bacteria or in other clinical situations.

The analysis of these factors in a group of patients with MRSA infections and a group of non-infected control patients randomly selected is thus relevant to isolate and identify risk factors for bacteraemia, or involved with more severe progression of the infection. The identification and measurement of these factors may be important elements in the design of effective intervention.

\section{MATERIALS AND METHODS}

The study was conducted in a 700-bed hospital school in Sao Paulo (Brazil) and included 60 case patients and 240 control patients.

The case group was composed of patients presenting blood culture positive for MRSA and who had signs and symptoms of infection, such as fever, chilling, clinical worsening or evidence of infection in target organs (pneumonia, surgical site), when blood was collected. Time of hospitalization, origin of the patient (home or hospital) and evidence of persistence of bloodstream infection were not considered. The cases were identified by analysis of the database of the microbiology laboratory. Information previous to bacteraemia and relevant for the investigation of risk factors was obtained from medical records and information referent to evolution to death or hospital discharge was prospectively obtained.

The 240 patients composing the control group (a 4:1 ratio in relation to the case group) were selected as the first four patients admitted to the hospital on the day a positive blood culture result was scored for a case patient. In cases where the patient was younger than 16 years, was hospitalized for less than $48 \mathrm{~h}$ or was admitted to psychiatric, ophthalmological or otolaryngological wards, the next patient was considered, so that the order of admission and of controls was concordant. The control patient was identified and prospectively followed, from admission until discharge from hospital or death.
The following information was collected and registered for case and control patients:

Identification data: name, hospital registration, date of birth, date and ward of admission, sex, underlying disease, date of nosocomial bloodstream infection episode, surgical procedure and severity of clinical condition according to the APACHE, ASA or McCabe criteria in cases of ICU, surgical or clinical profiles, respectively.

Risk factors: use of antimicrobial therapy and period during which the treatment was administered, presence of Central Vascular Catheter (CVC), presence of vesical probe, presence of mechanical ventilation, tracheostomy, admission and permanence in intensive care units, submission to hemodyalisis, peritoneal dialysis, corticotherapy or immunosuppressors, neutropenia, total length of hospitalization and evolution (discharge from hospital or death).

Patients were diagnosed with a wide range of diseases and were classified in 13 syndromes such as heart disease, neurologic disease, lung disease, kidney disease.

For a more appropriate analysis of the severity of the clinical cases and comparison of the three distinct scores used in this study (ASA, APACHE II and $\mathrm{McCabe}$ ), the patients were classified in two groups, according to presenting favourable evolution or rapidly fatal evolution. Patients with APACHE II $>10$, ASA $>2$ or $\mathrm{McCabe}=1$ were considered as presenting rapidly fatal evolution.

Indication and procedures for collection of blood from hospitalized patients for hemoculture, as well as laboratory routine activities, were not modified during the period of study. The growth of microorganisms was investigated with the BACTEC 9240 test. Identification and sensitivity tests were performed according to recommendations of the CLSI: Clinical and Laboratory Standards Institute.

Infection-related death was defined as that occurring during the first 14 days after bacteraemia was diagnosed and blood was collected for culture.

Statistical analysis: Data were analyzed using SPSS (version 11.0) and Epi-Info (version 3.3.2) software's. In university analysis, Student's t-test was used for quantitative variables and Pearson's qui-square or Fisher's exact test for discrete variables. To investigate the combined influence of the variables over the risk of bacteraemia or death, stepwise logistic regression models were adjusted for variables presenting $\mathrm{p}<0.1$ in university analysis. Results were expressed as Odds Ratio (OR) and the respective 95\% Confidence Intervals $(\mathrm{CI})$. 


\section{RESULTS}

The incidence of MRSA bacteraemia in the population investigated in this study was $1.99 / 1000$ hospital admissions, or 0.43 infections/1000 patients/day. The mortality rate of case patients, which we defined as deaths occurring within the first 14 days after bacteraemia was diagnosed, was 30\%. Mortality rate among controls was $5.38 \%$. The agent thus presented lethality (mortality among case patients less mortality among controls) of $24.6 \%$.

Mean age was 54.7 (21-88) years for case patients and $51.1(16-95)$ years for controls $(\mathrm{p}=0.19)$. Among case patients, $58.3 \%$ were male, whereas among controls $45.0 \%$ were male $(\mathrm{p}=0.06)$. Mean hospitalization time was 53.7 days for case patients, with a range of 1 to 258 days and 9 days for controls, with a range of 2 to 99 days $(\mathrm{p}<0.01)$. The mortality rate was $30 \%$ in the case group and $5.3 \%$ in the control group $(\mathrm{p}<0.01)$.

Multivariate analysis, after elimination of factors with no significance at the $\mathrm{p}<0.1$ level and analysis of the interaction among the remaining factors, showed that risk factors for bacteraemia were related to severity of the disease, when patients presented $\mathrm{McCabe}=1$, ASA $>2$ or APACHE II>10 (OR: 4.1; 95\% CI: 1.8-9.4) and presence of central venous catheter (OR: $7.3 ; 95 \%$ CI: 2.4-22.2). The only protective factor observed was having been submitted to surgery (OR: $0.2 ; 95 \%$ CI: 0.07-0.6). These results was shown in Table 1.

Table 1: Multivariate analysis of risk factor for bacteraemia

\begin{tabular}{lcll}
\hline Parameter & p-value & Odds ratio & $95 \%$ CI \\
\hline Age (years) & 0.678 & 1.00 & $0.98-1.03$ \\
Sex: male & 0.231 & 1.64 & $0.73-3.69$ \\
Rapidly fatal* & 0.001 & 4.15 & $1.82-9.47$ \\
Previous hospitalization & 0.978 & 1.01 & $0.40-2.55$ \\
Venous central catheter & 0.000 & 7.33 & $2.41-22.19$ \\
Vesical probe & 0.056 & 2.69 & $0.97-7.44$ \\
Corticotherapy & 0.113 & 1.97 & $0.85-4.59$ \\
Hemodialysis & 0.214 & 2.22 & $0.63-7.85$ \\
Intensive care unit & 0.464 & 0.66 & $0.22-1.99$ \\
Previous surgery & 0.005 & 0.21 & $0.07-0.63$ \\
\hline *ASA > 2 or McCabe $=1$ or Apache $>10$ & \\
& & & \\
Table 2: Multivariate analysis of risk factor for mortality & \\
\hline Parameter & p-value & Odds ratio & $95 \%$ CI \\
\hline Age (years) & 0.717 & 1.23 & $0.40-3.75$ \\
Sex: male & 0.000 & 1.08 & $1.03-1.12$ \\
Rapidly fatal* & 0.001 & 9.13 & $2.57-32.35$ \\
Previous surgery & 0.004 & 0.09 & $0.02-0.46$ \\
Venous central catheter & 0.722 & 0.75 & $0.15-3.64$ \\
Vesical probe & 0.100 & 3.23 & $0.79-13.10$ \\
Mechanical ventilation & 0.009 & 10.32 & $1.78-59.51$ \\
Intensive care unit & 0.952 & 0.96 & $0.23-3.87$ \\
Hemodialysis & 0.386 & 1.98 & $0.42-9.26$ \\
Corticotherapy & 0.001 & 9.96 & $2.69-36.78$ \\
\hline
\end{tabular}

Multivariate analysis of risk factors for mortality, after elimination of factors with no significance at the $\mathrm{p}<0.1$ level and analysis of the interaction among the remaining factors, showed significance for age (OR: 1.08; 95\% CI: 1.03-1.12), use of mechanical ventilation (OR: 10.32; 95\% CI: 1.78-59.51) and corticotherapy (OR: 9.9.96; 95\% CI: 2.69-36.78). Surgery was observed as a protective factor for mortality risk (OR: 0.09 ; 95\% CI: 0.02-0.46). The results was shown in Table 2.

\section{DISCUSSION}

The incidence of MRSA bacteraemia observed in the present study, 1.99/1000 hospital admissions, or 0.43 infections/1000 patients/day, was higher than previously reported. Cordova et al. described an incidence of $0.25 / 1000$ admissions $^{[13]}$, Jensen et al. ${ }^{[14]}$, in a study that did not discriminate between MRSA and Methicillin-Sensitive S. Aureus (MSSA), observed an incidence of $0.7 / 1000$ admissions and Tacconelli et al. ${ }^{[15]}$ observed 1.3 cases of MRSA/1000 admissions during the first $24 \mathrm{~h}$ after hospitalization.

Bloodstream MRSA infection is associated with high mortality rates, independent of the underlying disease $^{[16,17]}$. The mortality rate of $30 \%$ observed in the present study and the risk factors for bacteraemia such as use of central venous catheter, corticotherapy and clinical score of high severity, agree with results previously reported. The study by Cassettari et al. ${ }^{[18]}$, for instance, described a mortality rate associated to MRSA bacteraemia of $33 \%$ and corticotherapy, presence of central venous catheter, age older than 60 years and previous use of antibiotics, as associated risk factors.

The elements investigated as risk factors for bacteraemia were also analyzed as possible predictors of mortality by MRSA. The results showed older age, severity of the disease, use of mechanical ventilation and corticotherapy as risk factors for mortality and surgery as a protective factor.

More severe clinical conditions are expected to be associated to higher mortality rates and this association has been shown in previous studies. Gómez et al. ${ }^{[19]}$, for instance, observed by multivariate analysis that rapidly fatal disease and altered conscience level at onset, among other factors, are the main prognostic factors associated with mortality in patients with MRSA bacteraemia.

Other risk factors, such as age and immunosuppression, have also been previously reported. In a large study with 424 cases of $S$. aureus 
bacteremia performed in New Zealand ${ }^{[20]}$, older age than 60 years, feminine sex, diabetes, immunosuppression and lung as a source of bacteremia were identified as risk factors for mortality.

The present study, however, aimed at investigating the influence of the selection of the control group in the evaluation of risk factors for bacteremia and mortality associated to $S$. aureus infection. In most studies, sensitivity to methicillin is used as the major element to compare risk factors for case and control groups. We worked under the assumption that, by selecting for the control group patients without methicillin-sensitive $S$. aureus bacteremia, risk factors previously described could be confirmed of dissociated and other elements could be identified as risk factors. The large differences in disease severity observed between the case and control groups, however, influenced the comparison and introduced a bias in the study. The results might thus be due to the characteristics inherent to each group or, alternatively, to the difference in disease severity, so that risk factors might be related to higher disease severity presented by the case group. Similarly, the protective factor (surgery) might reflect a bias introduced by the criteria for selection of patients to be submitted to elective surgery.

The use of three different severity scores (ASA, APACHE II or McCabe), has also introduced a difficulty in the analysis of results. Nevertheless, the inclusion in the study of patients from ICU, as well as patients hospitalized for their clinical conditions or submitted to elective surgery, prevented the use of a single severity score. Our results, as a whole, showed moderate influence of control group selection, in comparison to studies in which methicillin-sensitive $S$. aureus infection is used as control.

\section{REFERENCES}

1. Chambers, H.F., 2001. The changing epidemiology of Staphylococcus aureus? Emerg. Infect. Dis., 7: 178-182.

http://www.cdc.gov/ncidod/eid/vol7no2/chambers. htm.

2. Lowy, F.D., 1998. Staphylococcus aureus infections. N. Engl. J. Med., 339: 520-532.

3. Hospital Infections Program, National Center for Infectious Diseases, Centers for Disease Control and Prevention, Public Health Service, U.S. Department of Health and Human Services. National Nosocomial Infections Surveillance (NNIS) system report: data summary from January 1990-May 1999, issued June 1999. Am. J. Infect. Control, 27: 520-532.
4. Vincent, J.L., D.J. Bihari and P.M. Suter et al., 1995. The prevalence of nosocomial infection in intensive care units in Europe. Results of the European Prnceevale of Infection in Intensive Care (EPIC) Study. JAMA, 274: 639-644. http://jama.amaassn.org/cgi/content/abstract/274/8/639

5. Eggimann, P. and D. Pittet, 2002. Overview of catheter-related infections with special emphasis on prevention based on educational programs. Clin. Microbiol. Infect., 8: 295-309. 10.1046/j.14690691.2002.00467.x

6. Groom, A.V., D.H. Wolsey and T.S. Naimi et al., 2001. Community-acquired methicillin-resistant Staphylococcus aureus in a rural American Indian community. JAMA, 286: 1201-1205. http://jama.ama-

assn.org/cgi/content/abstract/286/10/1201

7. Centers for Disease Control and Prevention, 200203. Outbreaks of community-associated methicillin-resistant Staphylococcus aureus infections-Los Angeles County, 52: 88.

8. Zinderman C.E., B. Conner and M.A. Malakooti et al., 2004. Community-acquired methicillinresistant Staphylococcus aureus among military recruits. Emerg. Infect. Dis., 10: 941-944. http://www.cdc.gov/ncidod/eid/vol10no5/030604.htm

9. Centers for Disease Control and Prevention. Methicillin-resistant Staphylococcus aureus among competitive sports participants--Colorado, Indiana, Pennsylvania and Los Angeles County, 2000-2003. MMWR Morb Mortal Wkly Rep., 52: 793-5.

10. Centers for Disease Control and Prevention. Public health dispatch:outbreaks of community-associated methicillin-resistant Staphylococcus aureus skin infections -- Los Angeles County, 2003-2003. MMWR Morb Mortal Wkly Rep., 23: 88.

11. Centers for Disease Control and Prevention. Four pediatric deaths from community-acquired methicillin-resistant Staphylococcus aureusMinnesota and North Dakota, 1997-1999. MMWR Morb Mortal Wkly Rep. 1999 48: 707-710.

12. Cosgrove, S.E., G. Sakoulas and E.N. Perencevich et al., 2003. Comparison of mortality associated with methicillin-resistant and methicillinsusceptible Staphylococcus aureus bacteremia:a meta-analysis. Clin. Infect. Dis., 36: 53-59. http://cat.inist.fr/?aModele $=$ afficheN\&cpsidt $=1446$ 7466. 
13. Cordova, S.P., C.H. Heathc and D.B. McGechied et al., 2004. Methicillin-resistant Staphylococcus aureus bacteraemia in Western Australian teaching hospitals, 1997-1999: risk factors, outcomes and implications for management. J. Hosp. Infect., 56: 22-28.

http://linkinghub.elsevier.com/retrieve/pii/S019567 0103004158.

14. Jensen, A.G., C.H. Wachmann and K.B. Poulsen et al., 1999. Risk factors for hospital-acquired Staphylococcus aureus bacteremia. Arch. Int. Med., 159: 1437-1444. http://archinte.amaassn.org/cgi/content/abstract/159/13/1437.

15. Tacconelli, E., L. Venkataraman and P.C. De Girolami et al., 2004. Methicillin-resistant Staphylococcus aureus bacteraemia diagnosed at hospital admission: distinguishing between community-acquired versus healthcare-associated strains. J. Antimicrob. Chemother., 53: 474-479. http://jac.oxfordjournals.org/cgi/content/abstract/53 /3/474.

16. Moreira, M., E.A. Medeiros and A.C. Pignatari et al., 1998. Effect of nosocomial bacteremia caused by oxacillin-resistant Staphylococcus aureus on mortality and length of hospitalization. Rev. Assoc. Med. Bras., 44: 263-268.

http://www.ncbi.nlm.nih.gov/pubmed/9852643.
17. Conterno, L.O., S.B. Wey and A. Castelo, 1998. Risk factors for mortality in Staphylococcus aureus bacteremia. Infect. Control. Hosp. Epidemiol., 19: 32-37. http://www.ncbi.nlm.nih.gov/pubmed/9475347

18. Cassettari, V.C., T. Strabelli and E.A. Medeiros, 2005. Staphylococcus aureus bacteremia: what is the impact of oxacillin resistance on mortality? Braz. J. Infect. Dis., 9: 70-76.

119.Gomez, J., E. Garcia-Vazquez and R. Banos et al., 2007. Predictors of mortality in patients with methicillin-resistant Staphylococcus aureus (MRSA) bacteraemia: the role of empiric antibiotic therapy. Eur. J. Clin. Microbiol. Infect. Dis., 26: 239-245.

20. Hill, P.C., M. Birch and S. Chambers et al., 2001. Prospective study of 424 cases of Staphylococcus aureus bacteraemia: determination of factors affecting incidence and mortality. Intern. Med. J., 31: 97-103. 